\title{
Development and Application of Mesoscopic CFD Methods for Compressible and Thermal Flows
}

\author{
Lian-Ping Wang \\ Chair Professor, Department of Mechanics and Aerospace Engineering \\ Southern University of Science and Technology \\ Shenzhen, Guangdong, China \\ wanglp@sustech.edu.cn
}

\begin{abstract}
Since the 1980s, direct numerical simulations have served as a vital research tool to probe flow structures and transport processes in complex flows such as thermal flows and turbulent flows. Most of these simulations were performed based on the continuum (conventional or macroscopic) Navier-Stokes equations. In recent years, mesoscopic methods based on the Boltzmann equation, such as the lattice Boltzmann method and gas kinetic schemes, have been developed and applied to these complex flows. In this talk, I will discuss our recent work on extending a specific mesoscopic method known as the discrete unified gas kinetic scheme (DUGKS) for simulations of compressible turbulence and compressible natural convection flows. My talk will cover the underlying theory, code implementation, and simulation results.
\end{abstract}

